\title{
Outcomes for Difficult-to-Wean Subjects After Cardiac Surgery
}

\author{
Guido Vagheggini MD, Eugenia Panait Vlad MD, Stefano Mazzoleni PhD, \\ Uberto Bortolotti MD, Fabio Guarracino MD, and Nicolino Ambrosino MD
}

\begin{abstract}
BACKGROUND: The need for prolonged mechanical ventilation (PMV) after cardiac surgery is still a common problem. We hypothesized that subjects who required PMV after a single surgery (either coronary artery bypass grafting or valve surgery) would have better outcomes than those who had experienced both revascularization and valve surgery. METHODS: We retrospectively analyzed the characteristics and outcomes for patients consecutively admitted to our weaning unit (WU) after cardiac surgery between December 2007 and August 2012. Subjects' data were analyzed according to the number of procedures. Group 1 included subjects who had undergone a single surgery (valvular replacement or myocardial revascularization). Group 2 included subjects who had undergone combined surgery (valvular replacement and myocardial revascularization). Data for PMV subjects who were transferred to the WU for reasons other than cardiac surgery in the same period are also reported. RESULTS: Of 584 PMV subjects admitted, 35 (5.9\%, 19 in group 1 and 16 in group 2) were referred after cardiac surgery. At WU admission, group 2 showed significantly more comorbidities and a greater clinical severity than group 1. Compared with group 1 , group 2 showed a significantly lower weaning rate $(43.7 \%$ vs $78.9 \%, P=.003)$ and a higher in-hospital mortality $(31.3 \%$ vs $5.3 \%, P=.04)$. The overall 6 -month survival for subjects of group 1 was $73.7 \%$ compared with $37.5 \%$ for subjects in group $2(P=.02)$. Adjusting for comorbidities and clinical severity, the difference between the 2 groups did not reach statistical significance for either the weaning rate or the overall 6-month survival. At discharge, health status, as assessed by means of functional independence measure, was significantly better in group 1 than in group 2 $(P=.035)$. CONCLUSIONS: These results suggest that patients needing PMV after combined cardiac surgery may suffer worse outcomes than those needing PMV after simple cardiac surgery. Key words: cardiac surgery; mechanical ventilation; respiratory failure; weaning; mechanical ventilation. [Respir Care 2015;60(1):56-62. () 2015 Daedalus Enterprises]
\end{abstract}

\section{Introduction}

Invasive mechanical ventilation requires admission to an ICU. Most subjects need only short periods of respiratory support, with a minority requiring prolonged mechanical ventilation (PMV). ${ }^{1}$ More patients who require mechanical ventilation are surviving, ${ }^{2}$ leading to a probable

\footnotetext{
Drs Vagheggini, Vlad, and Ambrosino are affiliated with the Pulmonary Rehabilitation, and Weaning Unit, Auxilium Vitae, Volterra; Drs Vlad, Mazzoleni, and Ambrosino are affiliated with the Rehabilitation Bioengineering Laboratory, Auxilium Vitae, Volterra; Dr Mazzoleni is affiliated with the BioRobotics Institute, Scuola Superiore Sant'Anna, Pisa; Dr Bortolotti is affiliated with the Cardio-Surgery Unit, University Hospital, Pisa; Dr Guarracino is affiliated with the Cardio-Surgery Intensive Care Unit, University Hospital, Pisa, Italy.
}

increase in PMV incidence 3 : up to $6 \%$ of ventilated ICU patients ${ }^{4,5}$ or $14 \%$ of patients starting weaning, ${ }^{6}$ with approximately $10 \%$ of these patients requiring tracheostomy. ${ }^{2}$ These patients have high resource utilization and relatively poor outcomes, especially in the elderly or those with comorbidities that may require long-term acute care facilities or specialized weaning units (WU). ${ }^{7-9}$

\footnotetext{
The authors have disclosed no conflicts of interest.

Correspondence: Nicolino Ambrosino MD, Pulmonary Rehabilitation and Weaning Unit, Auxilium Vitae, Borgo San Lazzero 5, 56048 Volterra (PI), Italy. E-mail: nico.ambrosino@gmail.com.
}

DOI: $10.4187 /$ respcare. 03315 
Despite advances in surgical techniques and anesthetic management, PMV after cardiac surgery such as coronary artery bypass grafting $(\mathrm{CABG})$ is still a relatively common occurrence, with a reported incidence between 2.9 and $8.6 \%$ when subjects need intubation and mechanical ventilation for a period longer than $1 \mathrm{~h}$ after completion of surgery. ${ }^{10-14}$ To the best of our knowledge, there are no outcome data for cardiac surgery subjects undergoing PMV with tracheostomy. ${ }^{1}$ We hypothesized that subjects who need PMV after a single surgery that is either CABG or valve surgery would have better outcomes than those subjects who have experienced both revascularization and valve surgery. The evaluated clinical outcomes were: rate of weaning and tracheostomy closure, ventilator dependence, health status, WU length of stay (LOS), location after discharge, in-hospital mortality, and 6-month mortality..

\section{Methods}

The study was performed in an 8-bed WU located inside a 20-bed pulmonary rehabilitation unit of a rehabilitation hospital, which is the referral center of a large geographic area for difficult-to-wean patients. It is staffed with a dedicated medical doctor from 8 AM to 8 PM, a 1:6 nurse to patient ratio (1 full-time nurse in the WU and 1 nurse shared with the pulmonary rehabilitation unit), a 24-hour on-duty doctor shared with the pulmonary rehabilitation unit, a daytime 1:6 respiratory therapist to patient ratio (with the same commitment proportion as for nurses). Psychological, speech, nutrition, and swallowing services are also available. Difficult-to-wean tracheostomized subjects are transferred from ICUs of other hospitals to the WU to undergo a program of progressive discontinuation from mechanical ventilation or to be discharged to a home program of long-term ventilatory assistance, if weaning from the ventilator fails, as previously described. ${ }^{9}$

\section{Subjects}

The protocol was approved by the ethics committee of University Hospital, Pisa. All subjects or their relatives gave written informed consent for the use of their data for research purposes.

We retrospectively analyzed the characteristics and outcomes of tracheostomized subjects consecutively admitted to the WU after cardiac surgery between December 2007 and August 2012 for a program of weaning from PMV. PMV was defined as $>7 \mathrm{~d}$ of mechanical ventilation after the first spontaneous breathing trial (SBT). ${ }^{1}$ Data for PMV subjects transferred to the WU for reasons other than cardiac surgery in the same period are also reported and compared with those for cardiac surgery subjects.

\section{QUICK LOOK}

\section{Current knowledge}

Mechanical ventilation following cardiac surgery is commonly of limited duration. Prolonged mechanical ventilation (PMV) following cardiac surgery is associated with significant morbidity and costs. The risk factors for PMV include emergency surgery, co-morbidities, and operative times.

\section{What this paper contributes to our knowledge}

The need for PMV following cardiac surgery was more common in subjects requiring both myocardial revascularization and valve replacement. After adjusting for comorbidities and illness severity, the differences in weaning rate and 6-month mortality between groups were not significant.

\section{Weaning Techniques}

In our WU, patients undergo weaning protocols either of progressively decreasing levels of pressure-support ventilation (PSV) or progressively longer periods of SBT as previously described. ${ }^{915-17}$ Physiotherapy is also performed according to accepted protocols. ${ }^{18,19}$ Tracheal cannula removal and tracheostomy closure are performed on an individual basis according to criteria described elsewhere. ${ }^{20}$

\section{Measurements}

Data were obtained from subjects' records and unit database. Survival data were obtained from the local healthcare system registry (ADTWeb ASL5, Pisa, Italy).

Age, gender, admission diagnoses, Acute Physiology and Chronic Health Evaluation (APACHE-II) ${ }^{21}$ and Simplified Acute Physiology Score (SAPS) II, 22 chronic comorbidities according to Charlson index ${ }^{23}$ (all these indexes are routinely recorded at WU admission), and previous LOS in the ICU of origin were collected. Clinical outcomes were: (1) weaning success, tracheal cannula removal and tracheostomy closure, and ventilator dependence; (2) health status by means of functional independence measure 24,25 ; (3) WU LOS; (4) location after discharge (home, nursing home, rehabilitation hospital, acute-care hospital); and (5) in-hospital and 6-month mortality.

Successful weaning was defined according to the National Association for Medical Direction of Respiratory Care consensus conference as the ability to breathe spontaneously for more than 7 consecutive days and to be discharged alive. ${ }^{26}$ Weaning time was defined as the time 
elapsed from WU admission to the first day of spontaneous breathing and is reported only for subjects considered weaned according to the above definition. ${ }^{26}$ Subjects still receiving mechanical ventilation within $35 \mathrm{~d}$ and subjects who died in hospital within $35 \mathrm{~d}$ were considered to have failed weaning. These subjects were considered censored at the end of the study period of $35 \mathrm{~d}$, and the weaning time was set equal to $35 \mathrm{~d}$. Alternatively, subjects who died in hospital were also considered as censored at the time of death, and the weaning time was defined as the time elapsed from WU admission to the time of death. ${ }^{27}$

Cardiac surgery subjects' data were analyzed according to the number of cardiac surgical procedures. Group 1 included subjects who underwent a single surgery (valvular replacement or myocardial revascularization). Group 2 included subjects who underwent more than one surgical procedure (eg, valvular replacement and $\mathrm{CABG}$ ).

\section{Statistical Analysis}

Data are shown as mean $\pm \mathrm{SD}$. Although this study addresses several outcome measures, only one was considered for sample size determination. Difference in weaning rate between 2 groups was the outcome selected for determining a minimum sample size. To ensure $70 \%$ power to detect a $35 \%$ difference in weaning ( $80 \%$ vs $45 \%$ ) as significant at the 0.05 level, 17 subjects/group were needed for the study.

Subjects' characteristics in both groups were compared by using chi-square test for nominal data and independent sample $t$ test or nonparametric Wilcoxon test for continuous data. Yates correction was applied in comparing frequencies in small groups. Paired $t$ test was used to compare admission versus discharge functional independence measure values. The Kaplan-Meier method was used to determine the probability of continuing mechanical ventilation and 6-month survival, and the log-rank test was used to compare groups.

The difference between groups was also evaluated by the Cox proportional hazard regression model adjusting for confounding covariates: Acute Physiology and Chronic Health Evaluation (APACHE-II), Simplified Acute Physiology Score (SAPS) II, and chronic comorbidities according to Charlson index.

All covariates and the group variable were inserted in an multivariate Cox regression model. Statistical significance was measured by the likelihood ratio chi-square (LR). The $\log -\log$ survival function was examined to verify that the proportionality assumption was met, and a test for the assumption of proportional hazards was performed by entering into the model an interaction term between variables and time. The hazard ratio (HR) with $95 \% \mathrm{CI}$ was reported. A 2 -sided $P$ value $<.05$ was considered as statistically significant. The statistical analysis was performed using JMP 4.00 statistical software (SAS Institute, Cary, North Carolina).

\section{Results}

Out of 584 PMV subjects admitted to the WU (337 males, age $70.7 \pm 11.7$ y) between December 2007 and August 2012, 35 (5.9\%) were referred after cardiac surgery. Nineteen subjects underwent a single surgery (group 1: 9 valve replacements [2 mitral, 7 aortic], 8 myocardial revascularizations, 1 atrial resection for neoplasm, and 1 rescue cardiac surgery after a complicated coronary angioplasty). Sixteen subjects underwent both myocardial revascularization and valvular surgery (group 2: 1 aortic plus mitral valve replacement, 11 aortic valve replacements, 1 mitral valve replacement, and 3 mitral valvuloplasty).

In the same period, 549 subjects who underwent noncardiac surgery were admitted to the WU. The causes of ICU admissions were: COPD, 150 (27.3\%); other respiratory diseases, 109 (19.8\%); non-cardiac surgery, 73 (13.3\%); neuromuscular diseases, 65 (11.8\%); severe brain injuries, 48 (8.7\%); trauma, $48(8.7 \%)$; heart failure, 43 (7.8\%); and other, 13 (2.4\%).

Demographic, physiological, and clinical characteristics of subjects at WU admission are shown in Table 1. There was no significant difference in any parameter between cardiac surgery and non-cardiac surgery subjects. Previous ICU LOS and time since tracheostomy were not different between groups 1 and 2. At admission, compared with group 1, group 2 subjects showed significantly more comorbidities as assessed by Charlson score, and a greater clinical severity as assessed by APACHE II score. Infection incidence at admission was not significantly different between groups $(63.1 \%$ and $87.5 \%$ in groups 1 and 2 , respectively, $P=.21$ ). During WU stay, 5 additional WUacquired infections were observed in each group.

\section{Outcome for Cardiac Surgery Versus Non-Cardiac Surgery Subjects}

Outcome measures in non-cardiac surgery and cardiac surgery subjects are shown in Table 2 . There was no significant difference in any outcome measure between the 2 populations.

\section{Outcome for Combined Versus Simple Cardiac Surgery Subjects}

Weaning. There was no significant difference between groups in weaning techniques used: progressively longer periods of SBT were used in 11 (57.9\%) and 10 (62.5\%), whereas progressive reduction in PSV level was used in 
Table 1. Demographic, Clinical, and Physiological Characteristics of Subjects at Admission

\begin{tabular}{|c|c|c|c|c|c|c|}
\hline & $\begin{array}{l}\text { Non-Cardiac Subjects } \\
\quad(n=549)\end{array}$ & $\begin{array}{l}\text { Cardiac Subjects } \\
\quad(n=35)\end{array}$ & $P$ & $\begin{array}{l}\text { Group 1 } \\
(n=19)\end{array}$ & $\begin{array}{l}\text { Group 2 } \\
(n=16)\end{array}$ & $P$ \\
\hline Males, $n$ & 319 & 17 & .55 & 7 & 10 & .24 \\
\hline Age, y & $70.5 \pm 11.8$ & $73.0 \pm 10.1$ & .16 & $71.0 \pm 12.2$ & $75.5 \pm 6.5$ & .18 \\
\hline Time from tracheostomy, $\mathrm{d}$ & $38.2 \pm 51.6$ & $39.8 \pm 29.5$ & .77 & $40.8 \pm 31.1$ & $38.7 \pm 28.6$ & .84 \\
\hline ICU stay, d & $46.9 \pm 44.2$ & $56.5 \pm 32.0$ & .09 & $55.2 \pm 33.2$ & $58.1 \pm 31.7$ & .80 \\
\hline SAPS II & $36.2 \pm 10.2$ & $35.9 \pm 8.6$ & .84 & $33.4 \pm 7.4$ & $38.9 \pm 9.2$ & .07 \\
\hline SAPS II predicted mortality, $\%$ & $24.8 \pm 16.1$ & $28.1 \pm 14.1$ & .18 & $26.0 \pm 12.3$ & $30.5 \pm 15.5$ & .36 \\
\hline Charlson index & $6.1 \pm 2.6$ & $6.8 \pm 2.7$ & .14 & $5.7 \pm 2.1$ & $8.1 \pm 2.7$ & .008 \\
\hline APACHE II score & $13.7 \pm 5.4$ & $13.5 \pm 5.8$ & .84 & $11.4 \pm 4.9$ & $16.1 \pm 6.0$ & .02 \\
\hline $\mathrm{P}_{\mathrm{aO}_{2}} / \mathrm{F}_{\mathrm{IO}_{2}}, \mathrm{~mm} \mathrm{Hg}$ & $245.0 \pm 86.3$ & $242.2 \pm 88.1$ & .86 & $232.5 \pm 86.3$ & $253.7 \pm 91.4$ & .50 \\
\hline $\mathrm{P}_{\mathrm{aCO}_{2}}, \mathrm{~mm} \mathrm{Hg}$ & $47.7 \pm 20.5$ & $45.4 \pm 11.2$ & .27 & $43.4 \pm 12.8$ & $47.8 \pm 8.8$ & .25 \\
\hline $\mathrm{pH}$ & $7.40 \pm 0.1$ & $7.42 \pm 0.06$ & .07 & $7.44 \pm 0.05$ & $7.41 \pm 0.08$ & .27 \\
\hline \multicolumn{7}{|c|}{$\begin{array}{l}\text { Values are mean } \pm \text { SD. } \\
\text { SAPS II = Simplified Acute Physiology Score II } \\
\text { APACHE II = Acute Physiology and Chronic Hea }\end{array}$} \\
\hline
\end{tabular}

Table 2. Outcome Measures

\begin{tabular}{lccc}
\hline \hline \multicolumn{1}{c}{ Location } & $\begin{array}{c}\text { Non-Cardiac } \\
\text { Subjects }\end{array}$ & $\begin{array}{c}\text { Cardiac } \\
\text { Subjects }\end{array}$ & $P$ \\
\hline Weaning unit stay (mean \pm SD), d & $27.3 \pm 16.8$ & $26.8 \pm 11.3$ & .81 \\
Weaning success, \% & 57.4 & 65.7 & .72 \\
Tracheostomy closure, \% & 60.3 & 86.9 & .33 \\
In-hospital mortality, \% & 12.7 & 17.1 & .69 \\
Home mechanical ventilation, \% & 17.1 & 13.5 & .78 \\
\hline
\end{tabular}

$42.1 \%$ and $37.5 \%$ of group 1 and group 2 subjects, respectively $(P=.38)$.

Twenty-three of 35 (65.7\%) cardiac surgery subjects were weaned. Six subjects $(17.1 \%)$ needed home invasive mechanical ventilation for longer than $12 \mathrm{~h} / \mathrm{d}$ at discharge from WU, whereas 6 died in the hospital. The only significantly different admission characteristic between weaned and unweaned subjects was SAPS II $(33.8 \pm 8.1$ vs $40.4 \pm 8.7, P=.05)$.

Figure 1 shows the probability of continuing mechanical ventilation for the 2 groups: compared with group 1 , group 2 showed a significant lower weaning rate $(84.2 \%$ vs $43.7 \%$, respectively, $\log$-rank $P=.003$ ). In weaned subjects, the weaning time was significantly different between groups: $3.1 \pm 4.2$ (range $1-13 \mathrm{~d}$ ) in group 1 and $9.1 \pm 8.3$ (range 1-24 d) in group 2 (Wilcoxon test $P=.04$ ). However, adjusting for the SAPS II score, APACHE II score, and Charlson score by the Cox model, the weaning rate was not significantly higher in group 1 than in group 2 $(\mathrm{HR}=2.6,95 \% \mathrm{CI}=0.99-7.5, P=.05)$.

This result was also confirmed when subjects who died in hospital were considered as censored at the time of death $(\mathrm{HR}=2.5,95 \% \mathrm{CI}=0.98-7.3, P=.05)$.

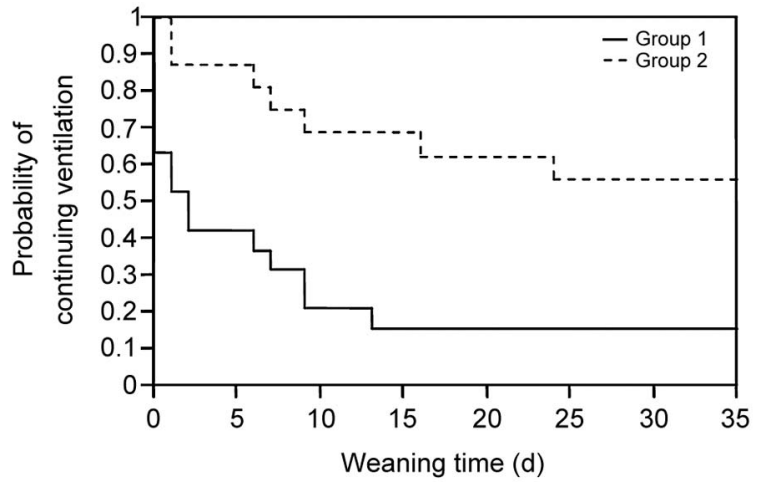

Fig. 1. Probability of continuing mechanical ventilation.

Tracheostomy closure. Fourteen out of $16(87.5 \%)$ and 6 out of $7(85.7 \%)$ weaned subjects of group 1 and group 2 , respectively, underwent tracheal cannula removal and tracheostomy closure, without any statistically significant difference between groups $(P=.91)$.

Mortality. Four subjects died in the WU as a result of septic (3 subjects) and cardiogenic shock (1 subject). Two additional subjects were transferred to an ICU for complications (hemoptysis, gastric perforation) requiring procedures not available in the WU, and subsequently died. Overall WU and in-hospital mortality rates were therefore $11.4 \%$ and $17.1 \%$, respectively, which is lower than the admission SAPS II-based predicted mortality rate (Table 1). A significantly higher in-hospital mortality was observed in group 2 compared with group 1 (31.3\% vs 5.3\%, $P=.042)$ : the mortality rate was lower than the admission SAPS II-based predicted mortality rate only in group 1 but not in group 2 (Table 1). 


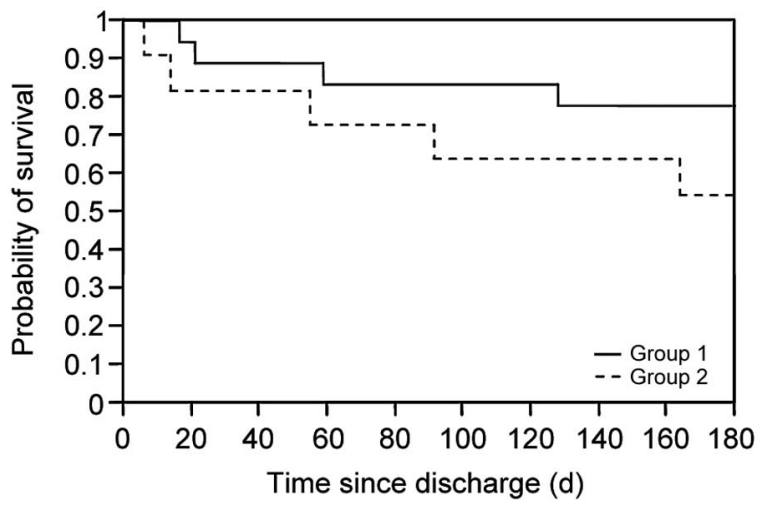

Fig. 2. Kaplan-Meyer 6-month survival curves of discharged subjects.

Figure 2 shows the post-discharge 6-month survival probability curves. Group 1 showed a statistically nonsignificant better survival rate. Indeed, 6 months after discharge 14 out of $18(77.8 \%)$ and 6 out of $11(54.5 \%)$ discharged subjects from group 1 and group 2 , respectively $(P=.37)$, were still alive. The overall 6-month survival rate of subjects admitted to our WU of group 1 was $73.7 \%$ compared with $37.5 \%$ of subjects of group 2 (log-rank $P=.02$ ). Adjusting for the SAPS II score, APACHE II score, and Charlson score by the Cox model, the survival rate was not significantly different between group 1 and group 2 $(\mathrm{HR}=1.6,95 \% \mathrm{CI}=0.4-6.2, P=.48)$.

Health status. Admission health status as assessed by means of functional independence measure was not statistically different $(39.9 \pm 14.2$ and $30.5 \pm 14.2$ in group 1 and 2 , respectively, $P=.06$ ) and improved significantly in group 1 (to $68.1 \pm 32.4, P<.001$ ), but nonsignificantly in group 2 (to $45.1 \pm 15.3, P=.06$ ); therefore, at discharge, health status was significantly better in group 1 than in group $2(P=.04)$.

\section{Final Destination}

Location after discharge is shown in Table 3. No significant difference was found between cardiac surgery and non-cardiac surgery subjects or between cardiac surgery groups 1 and 2.

\section{Discussion}

This retrospective study shows that admission to a WU results in weaning from PMV for most cardiac surgery subjects. Combined cardiac surgery is associated with lower weaning success rates, worse health status, and lower 6-month survival rates than single cardiac surgery.

Prolonged ICU stay by cardiac surgery subjects often results in a poorer clinical course and decreased survival after discharge. Data on clinical outcomes and prognosis are limited. Prolonged mechanical ventilation has been variously defined, $1,17,26,28-30$ and, despite advances in surgical techniques and anesthetic management, continues to be a relatively common problem after cardiac surgery such as $\mathrm{CABG}$, with a reported incidence between $2.9 \%$ and $8.6 \% .^{10-14}$ In this report, successful weaning was defined as the ability to breathe spontaneously for more than 7 consecutive days. ${ }^{26}$ Proposed definitions of weaning success for these difficult-to-wean subjects have included $48 \mathrm{~h}$, $7 \mathrm{~d}$, or $14 \mathrm{~d}$ without ventilatory support, and freedom from ventilator support at the time of hospital discharge. 1,17,26,28 Such differences in weaning success definition may be related to differences in patient population, discharge criteria, and institution-specific characteristics. ${ }^{26}$

Similarly, hospital mortality and LOS are widely variable. ${ }^{29}$ In our subjects, the overall WU mortality rate was $11.4 \%$, which is lower than the SAPS II predicted mortality rate. Subjects undergoing single cardiac surgery showed lower in-hospital and 6-month mortality rates than combined surgery subjects. As a matter of fact, in-hospital mortality rate was lower than the admission SAPS II-based predicted mortality rate in group 1 but not in group 2. A recent retrospective study reports that the in-hospital and 2 -y mortality rate were $16.1 \%$ and $42.6 \%$, respectively, in subjects needing mechanical ventilation for more than $7 \mathrm{~d}$ after cardiac surgery. ${ }^{31}$

When evaluating outcomes, it is necessary to consider the severity of subjects' medical status at admission. The severity of medical issues for the subjects of group 2 is demonstrated by long previous ICU stay, by mean APACHE II score of 16, and mean number of patient comorbidities. Furthermore, adjusting for the SAPS II score, APACHE II score, and Charlson score by the Cox model, the weaning rate was still higher (although at the limits of statistical significance) in group 1 than in group 2 and the survival rate was not significantly different between group 1 and group 2. Therefore, the difference in outcomes may be related more to the greater severity of underlying illness than to the type of surgery per se.

The mean weaning success rate of all cardiac surgery subjects in our study was similar to that of non-cardiac surgery PMV subjects admitted to our WU, being significantly higher in those subjects undergoing single cardiac surgery. In our WU, weaning protocols are used, namely, a strategy of progressive reduction of PSV or progressively longer periods of SBT. These techniques were not applied differently in either group; therefore, the difference in weaning success rate cannot be explained on the basis of different weaning techniques. Indeed, no significant difference in weaning success and mortality rate, duration of ventilatory assistance, WU, or total hospital LOS was reported between these 2 weaning techniques in difficult-to-wean subjects. ${ }^{17}$ The application of a well-de- 
Table 3. Location of Subjects Discharged From Weaning Unit

\begin{tabular}{lccccr}
\hline \hline \multicolumn{1}{c}{ Location } & $\begin{array}{c}\text { Non-Cardiac } \\
\text { Subjects }\end{array}$ & $\begin{array}{c}\text { Cardiac } \\
\text { Subjects }\end{array}$ & $P$ & Group 1 & Group 2 \\
\hline Home, $n(\%)$ & $278(50.6)$ & $15(48.4)$ & .73 & $10(55.5)$ & $5(38.5)$ \\
Nursing home, $n(\%)$ & $64(11.7)$ & $3(9.7)$ & .83 & $1(5.6)$ & $2(15.4)$ \\
Rehabilitation hospital, $n(\%)$ & $112(20.4)$ & $10(32.2)$ & .48 & $6(33.3)$ & $4(30.7)$ \\
Acute-care hospital, $n(\%)$ & $35(6.4)$ & $3(9.7)$ & .90 & $1(5.6)$ & .88 \\
\hline
\end{tabular}

fined protocol, independent of the mode used, may result in better outcomes than uncontrolled clinical practice. ${ }^{17,32}$

The need for tracheostomy may complicate cardiac surgery with an incidence ranging from $3.9 \%$ to $5.7 \%$ according to the type of surgery, and an increased risk of mortality, especially in the elderly. ${ }^{33}$ At least $85 \%$ of the weaned subjects of our study underwent cannula removal and tracheostomy closure. The clinical relevance of cannula removal at discharge is supported by a study showing that lack of decannulation of conscious tracheostomized subjects before ICU discharge to the general ward was associated with higher mortality. ${ }^{34}$ As a matter of fact, it has been shown that early tracheotomy provided no benefit in terms of mechanical ventilation and hospital LOS, rates of mortality or infectious complications, and long-term health status for subjects who require PMV after cardiac surgery. ${ }^{10}$ Furthermore, it has been reported that, in cardiac surgery subjects, tracheostomy is an independent risk factor for sternal wound infections..$^{35}$

An important component of our weaning program is the availability of physiotherapy. It has been demonstrated that early physiotherapy results in benefits in critical subjects in the ICU. $18,19,36,37$

Finally, an interesting result of our study is the destination of subjects discharged from the WU. The majority of subjects of both groups were able to be discharged home (Table 2). Our results are in agreement with those of a single-hospital, retrospective study, ${ }^{38}$ which followed 590 cardiac surgery subjects for at least $1 \mathrm{y}$ after discharge. At hospital discharge, $84.4 \%$ of subjects went home, $3.7 \%$ to rehabilitation program, $7.5 \%$ to skilled nursing facilities, and $4.4 \%$ to a longterm acute care facility. In that study, need for PMV decreased the odds of being home and alive at $1 \mathrm{y}$ by $67 \%$.

This study evaluated a 5-y period, during which treatment modalities (eg, weaning techniques, physiotherapy, and medical protocols) did not substantially change. One can argue that expertise of the caring team might have improved. This could be supported by the observation that the weaning rate was, although nonsignificantly, greater in those subjects admitted in the period 2011-2012 than in the previous period, 2007-2010, although Charlson index of comorbidities but not SAPS II or APACHE II scores or other demographic, anthropometric, or clinical characteristics differed in subjects who were admitted in the earlier observation time.

\section{Limitations of the Study}

This is a retrospective observational study; therefore, results must be considered with caution. Nevertheless, in our WU, clinical protocols and guidelines of the different actions remained comparable throughout the entire period of the study. Furthermore, a retrospective study may be considered as more representative of real-life management than a controlled prospective study.

The relatively small sample size may have induced some beta error, especially in evaluating the difference in 6-month survival rates. However, the same small sample size strengthens the message when differences are significant. Nevertheless, due to small sample size, this study did not evaluate the effect of on- or off-pump (cardiopulmonary bypass) on outcome. It has been shown that off-pump was associated with reduced comorbidities in CABG. ${ }^{39}$

\section{Conclusions}

With the above limitations, these results suggest that patients needing PMV after combined cardiac surgery may suffer from worse outcomes than after simple cardiac surgery. Whether this result must be ascribed to complexity of surgery rather than to more severe baseline clinical conditions remains to be elucidated. Further studies are needed to verify these data and identify further outcome predictors.

\section{ACKNOWLEDGMENTS}

We thank Professor Giuseppe Rossi PhD, biostatistician (Unit of Epidemiology and Biostatistics, Institute of Clinical Physiology, National Research Council, Pisa, Italy), for statistical advice and revision.

\section{REFERENCES}

1. Boles JM, Bion J, Connors A, Herridge M, Marsh B, Melot C, et al. Weaning from mechanical ventilation; statement of the Sixth International Consensus Conference on Intensive Care Medicine. Eur Respir J 2007;29(5):1033-1056.

2. Esteban A, Frutos-Vivar F, Muriel A, Ferguson ND, Peñuelas O, Abraira V, et al. Evolution of mortality over time in patients receiving mechanical ventilation. Am J Respir Crit Care Med 2013;188(2): 220-230.

3. Zilberberg MD, de Wit M, Pirone JR, Shorr AF. Growth in adult prolonged acute mechanical ventilation: implications for healthcare delivery. Crit Care Med 2008;36(5):1451-1455. 


\section{WeANing in CARDiac SuRgery}

4. Lone NI, Walsh TS. Prolonged mechanical ventilation in critically ill patients: epidemiology, outcomes and modelling the potential cost consequences of establishing a regional weaning unit. Critical Care 2011;15:R102.

5. Peñuelas O, Frutos-Vivar F, Fernández C, Anzueto A, Epstein SK, Apezteguía C, et al. Characteristics and outcomes of ventilated patients according to time to liberation from mechanical ventilation. Am J Respir Crit Care 2011;184(4):430-437.

6. Funk GC, Anders S, Breyer MK, Burghuber OC, Edelmann G, Heindl $\mathrm{W}$, et al. Incidence and outcome of weaning from mechanical ventilation according to new categories. Eur Respir J 2010;35(1):88-94.

7. Cox CE, Carson SS. Medical and economic implications of prolonged mechanical ventilation and expedited post-acute care. Semin Respir Crit Care Med 2012;33(4):357-361.

8. Ambrosino N, Gabbrielli L. The difficult-to-wean patient. Expert Rev Respir Med 2010;4(5):685-692

9. Carpenè N, Vagheggini G, Panait E, Gabbrielli L, Ambrosino N. A proposal of a new model for long-term weaning: respiratory intensive care unit and weaning center. Respir Med 2010;104(10):1505-1511.

10. Trouillet JL, Combes A, Vaissier E, Luyt CE, Ouattara A, Pavie A, Chastre J. Prolonged mechanical ventilation after cardiac surgery: outcome and predictors. J Thorac Cardiovasc Surg 2009;138(4):948953

11. Habib RH, Zacharias A, Engoren M. Determinants of prolonged mechanical ventilation after coronary artery bypass grafting. Ann Thorac Surg 1996;62(4):1164-1171.

12. Canver CC, Chanda J. Intraoperative and postoperative risk factors for respiratory failure after coronary bypass. Ann Thorac Surg 2003; 75(3):853-857; discussion 857-858.

13. Spivack SD, Shinozaki T, Albertini JJ, Deane R. Preoperative prediction of postoperative respiratory outcome: coronary artery bypass grafting. Chest 1996;109(5):1222-1230.

14. Légaré JF, Hirsch GM, Buth KJ, MacDougall C, Sullivan JA. Preoperative prediction of prolonged mechanical ventilation following coronary artery bypass grafting. Eur J Cardiothorac Surg 2001;20(5): 930-936.

15. Esteban A, Frutos F, Tobin MJ, Alía I, Solsona JF, Valverdú I, et al. A comparison of four methods of weaning from mechanical ventilation. N Engl J Med 1995;332(6):345-350.

16. Brochard L, Rauss A, Benito S, Conti G, Mancebo J, Rekik N, et al. Comparison of three methods of gradual withdrawal from ventilatory support during weaning from mechanical ventilation. Am J Respir Crit Care Med 1994;150(4):896-903.

17. Vitacca M, Vianello A, Colombo D, Clini E, Porta R, Bianchi L, et al. Comparison of two methods for weaning patients with chronic obstructive pulmonary disease requiring mechanical ventilation for more than 15 days. Am J Respir Crit Care Med 2001;164(2):225230.

18. Gosselink R, Bott J, Johnson M, Dean E, Nava S, Norrenberg M, et al. Physiotherapy for adult patients with critical illness: recommendations of the European Respiratory Society and European Society of Intensive Care Medicine Task Force on physiotherapy for critically ill patients. Intensive Care Med 2008;34(7):1188-1199.

19. Hanekom S, Gosselink R, Dean E, van Aswegen H, Roos R, Ambrosino N, Louw Q. The development of a clinical management algorithm for early physical activity and mobilization of critically ill patients: synthesis of evidence and expert opinion and its translation into practice. Clin Rehabil 2011;25(9):771-787.

20. Marchese S, Corrado A, Scala R, Corrao S, Ambrosino N. Tracheostomy in patients with long-term mechanical ventilation: a survey. Respir Med 2010;104(5):749-753.

21. Knaus WA, Draper EA, Wagner DP, Zimmerman JE. Apache II: a severity of disease classification system. Crit Care Med 1985;13(10): 818-829.
22. Le Gall JR, Lemeshow S, Saulnier F. A new Simplified Acute Physiology Score (SAPS II) based on a European/North American multicenter study. JAMA 1993;270(24):2957-2963.

23. Charlson M, Szatrowski TP, Peterson J, Gold J. Validation of a combined comorbidity index. J Clin Epidemiol 1994;47(11):12451251.

24. Montagnani G, Vagheggini G, Panait Vlad E, Berrighi D, Pantani L, Ambrosino N. Use of the functional independence measure in people for whom weaning from mechanical ventilation is difficult. Phys Ther 2011;91(7):1109-1115.

25. Tesio L. Functional assessment in rehabilitative medicine: principles and methods. Eur Medicophys 2007;43(4):515-523.

26. MacIntyre NR, Epstein SK, Carson S, Scheinhorn D, Christopher K, Muldoon S, National Association for Medical Direction of Respiratory Care. Management of patients requiring prolonged mechanical ventilation: report of a NAMDRC consensus conference. Chest 2005; 128(6):3937-3954.

27. Ranganathan P, Pramesh CS. Censoring in survival analysis: potential for bias. Perspect Clin Res 2012;3(1):40.

28. Scheinhorn DJ, Chao DC, Stearn-Hassenpflug M, LaBree LD, Heltsley DJ. Post-ICU mechanical ventilation: treatment of 1,123 patients at a regional weaning center. Chest 1997;111(6):1654-1659.

29. Pilcher DV, Bailey MJ, Treacher DF, Hamid S, Williams AJ, Davidson AC. Outcomes, cost and long term survival of patients referred to a regional weaning centre. Thorax 2005;60(3):187-192.

30. Gracey DR, Viggiano RW, Naessens JM, Hubmayr RD, Silverstein MD, Koenig GE. Outcomes of patients admitted to a chronic ventilator-dependent unit in an acute-care hospital. Mayo Clin Proc 1992; 67(2):131-136

31. Joskowiak D, Kappert U, Matschke K, Tugtekin S. Prolonged intensive care unit stay of patients after cardiac surgery: initial clinical results and follow-up. Thorac Cardiovasc Surg 2013 Jun 12;[Epub ahead of print]

32. Blackwood B, Alderdice F, Burns K, Cardwell C, Lavery G, O'Halloran P. Use of weaning protocols for reducing duration of mechanical ventilation in critically ill adult patients: Cochrane systematic review and meta-analysis. BMJ 2011;342:c7237.

33. Kamiya H, Tanzeem N, Akhyari P, Pedraza A, Kallenbach K, Lichtenberg A, Karck M. Impact of severe postoperative complications after cardiac surgery on mortality in patients aged over 80 years. Ann Thorac Cardiovasc Surg 2013 Jul 31; [Epub ahead of print]

34. Martinez GH, Fernandez R, Casado MS, Cuena R, Lopez-Reina P, Zamora S, Luzon E. Tracheostomy tube in place at intensive care unit discharge is associated with increased ward mortality. Respir Care 2009;54(12):1644-1652.

35. Sun L, Boodhwani M, Baer H, McDonald B. The association between tracheostomy and sternal wound infection in postoperative cardiac surgery patients. Can J Anaesth 2013;60(7):684-691.

36. Schweickert WD, Pohlman MC, Pohlman AS, Nigos C, Pawlik AJ, Esbrook CL, et al. Early physical and occupational therapy in mechanically ventilated, critically ill patients: a randomised controlled trial. Lancet 2009;373(9678):1874-1882.

37. Ambrosino N, Venturelli E, Vagheggini G, Clini E. Rehabilitation, weaning and physical therapy strategies in the chronic critically ill patients. Eur Respir J 2012;39(2):487-492.

38. Edgerton JR, Herbert MA, Mahoney C, Armstrong D, Dewey TM, Holper E, et al. Long-term fate of patients discharged to extended care facilities after cardiovascular surgery. Ann Thorac Surg;96(3): 871-877.

39. Polomsky M, He X, O’Brien SM, Puskas JD. Outcomes of off-pump versus on-pump coronary artery bypass grafting: impact of preoperative risk. J Thorac Cardiovasc Surg 2013;145(5):1193-1198. 